\title{
ACCOUNTING FOR AZIMUTH AMBIGUITIES IN INTERFEROMETRIC PERFORMANCE ANALYSIS
}

\author{
Michelangelo Villano, Gerhard Krieger \\ German Aerospace Center (DLR), Microwaves and Radar Institute
}

\begin{abstract}
Azimuth ambiguities affect the interferometric performance of SAR systems, causing a bias in the interferometric phase and a modulation of the interferometric coherence, as also visible in some TanDEM-X interferograms. This paper provides an explanation for this phenomenon and derives the analytical expressions for the phase bias and the coherence, resorting to the interferogram statistics for jointly circular Gaussian processes. The impact of azimuth ambiguities on the overall system performance is then considered. Plots are provided, which display the standard deviation of the phase bias, as well as the expected value and the standard deviation of the coherence loss component due to azimuth ambiguities. These plots can be useful for interferometric performance analysis.
\end{abstract}

Index Terms - Azimuth ambiguities, interferometry, microwave remote sensing, synthetic aperture radar (SAR)

\section{INTRODUCTION}

Azimuth ambiguities arise in synthetic aperture radar (SAR) images from finite sampling of the Doppler spectrum at the pulse repetition frequency (PRF). Since the spectrum repeats at PRF intervals, the signal components outside this frequency interval fold back into the main part of the spectrum [1].

The impact of azimuth ambiguities on interferometric performance is usually condensed in a coherence loss component $\gamma_{A m b, A z}[2]$, given by

$$
\gamma_{A m b, A z}=\frac{1}{1+A A S R}
$$

where $A A S R$ is the azimuth ambiguity-to-signal ratio. In this respect, azimuth ambiguities are considered in the same way as thermal noise.

Fig. 1 displays a detail of a TanDEM-X interferogram acquired over the Franz Josef Land, an archipelago located in the far north of Russia. In the top left-hand part, some sea ice, which surrounds the islands of the archipelago, can be distinguished. In this region, an unexpected coherence modulation can be observed, for which an explanation was not clear at the beginning. Taking a look to the interferometric phase, it can be noticed that the pattern of the bottom part of the image is somehow replicated in the sea ice region. The relative displacement of such a replica and the considerable backscatter difference between the two areas suggest that this effect may be due to azimuth ambiguities. Therefore, not only are azimuth ambiguities responsible for a coherence loss component, but they could also determine a significant phase bias, as well as a coherence modulation. A much deeper analysis of this phenomenon may be conducted by modelling the interferogram affected by azimuth ambiguities as the sum of interfering components and deriving its statistics [3].
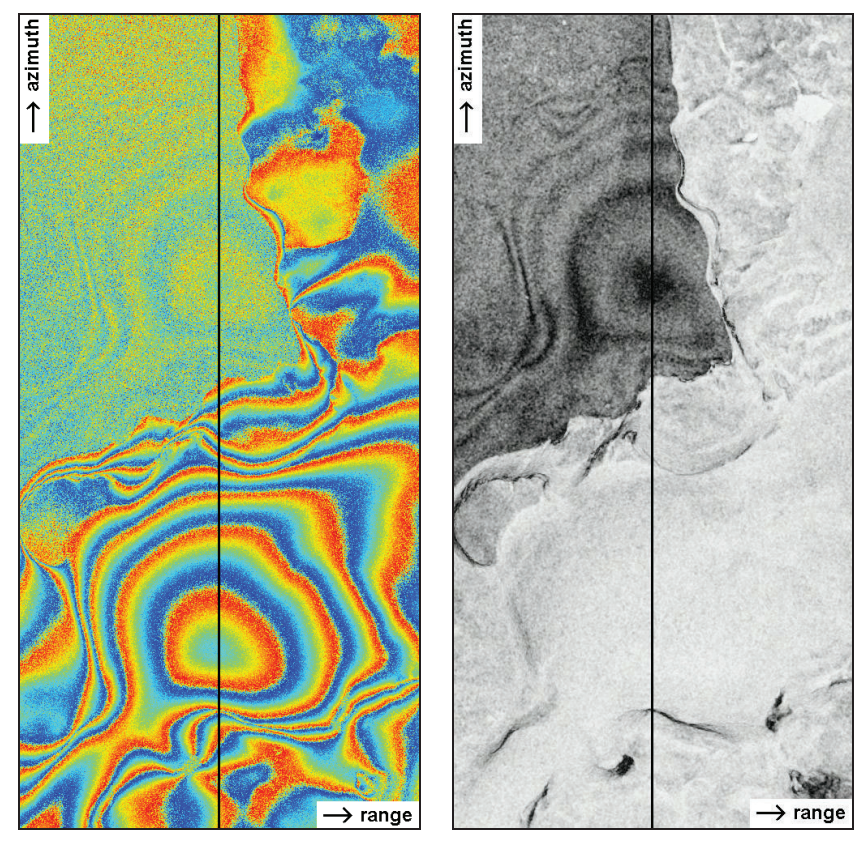

Fig. 1 Detail of a TanDEM-X interferogram affected by azimuth ambiguities: interferometric phase (left) and coherence (right).

\section{STATISTICS OF INTERFEROGRAMS AFFECTED BY AZIMUTH AMBIGUITIES}

Let $u[x, y]$ be a single-look complex (SLC) SAR image. $u[x, y]$ can be written as the sum of an ambiguity-free signal $m[x, y]$, from now on referred to as main signal, and a signal due to the azimuth ambiguity $a[x, y]$, from now on referred to as ambiguity signal

$u[x, y]=m[x, y]+a[x, y]$

We assume that $m[x, y]$ and $a[x, y]$ are statistically independent circularly symmetric complex Gaussian random variables with zero means and variances equal to $P_{m}$ and $P_{a}$, respectively. As a consequence, $u[x, y]$ is a circularly symmetric complex Gaussian random variable with zero mean and variance $\left(P_{m}+P_{a}\right)$.

In an interferometric scenario, both master and slave images, $u_{1}[x, y]$ and $u_{2}[x, y]$, are affected by ambiguities 
$u_{1}[x, y]=m_{1}[x, y]+a_{1}[x, y]$

$u_{2}[x, y]=m_{2}[x, y]+a_{2}[x, y]$

$m_{1}[x, y]$ and $m_{2}[x, y]$ are characterised by a variance or power equal to $P_{m}$ and are in general correlated, where $\gamma_{m}=\left|\gamma_{m}\right| \exp \left(\mathrm{j} \phi_{0 m}\right)$ is their complex correlation coefficient. $a_{1}[x, y]$ and $a_{2}[x, y]$ are characterised by a variance or power equal to $P_{a}$ and are also in general correlated, where $\gamma_{a}=\left|\gamma_{a}\right| \exp \left(\mathrm{j} \phi_{0 a}\right)$ is their complex correlation coefficient. $m_{k}[x, y], k=1,2$, and $a_{k}[x, y], \mathrm{k}=1,2$, are instead uncorrelated.

If an interferogram is formed from $u_{1}[x, y]$ and $u_{2}[x, y]$, four components, denoted as $v_{1}[x, y], v_{2}[x, y], v_{3}[x, y]$, and $v_{4}[x, y]$, arise

$$
\begin{aligned}
& v[x, y]=u_{1}[x, y] u_{2}^{*}[x, y]= \\
& \left(m_{1}[x, y]+a_{1}[x, y]\right)\left(m_{2}[x, y]+a_{2}[x, y]\right)^{*}= \\
& =m_{1}[x, y] m_{2}^{*}[x, y]+m_{1}[x, y] a_{2}^{*}[x, y]+ \\
& a_{1}[x, y] m_{2}^{*}[x, y]+a_{1}[x, y] a_{2}^{*}[x, y]= \\
& v_{1}[x, y]+v_{2}[x, y]+v_{3}[x, y]+v_{4}[x, y]
\end{aligned}
$$

The contribution $v_{1}[x, y]$ is the interferogram formed from the two main signals, $m_{1}[x, y]$ and $m_{2}[x, y]$, the contribution $v_{4}[x, y]$ is the interferogram formed from the two ambiguity signals, $a_{1}[x, y]$ and $a_{2}[x, y]$, while the contributions $v_{2}[x, y]$ and $v_{3}[x, y]$ are the interferograms formed by combining the main and ambiguity signals.

The processes $m_{1}[x, y]$ and $m_{2}[x, y]$ can be assumed to be jointly circular Gaussian. The processes $a_{1}[x, y]$ and $a_{2}[x, y]$ can also be assumed to be jointly circular Gaussian. Being $\mathbf{u}[x, y]=$ $\left[u_{1}[x, y], u_{2}[x, y]\right]$ a linear functional of jointly circular Gaussian processes, namely the sum of $\mathbf{m}[x, y]=\left[m_{1}[x, y], m_{2}[x, y]\right]$ and $\mathbf{a}[x, y]$ $=\left[a_{1}[x, y], a_{2}[x, y]\right], u_{1}[x, y]$ and $u_{2}[x, y]$ are also jointly circular Gaussian [4].

Being $u_{1}[x, y]$ and $u_{2}[x, y]$ jointly circular Gaussian processes, the interferogram $v[x, y]$ can be statistically characterised in terms of the joint probability density function (PDF) of magnitude $|v|$ and phase $\phi[5]$

$$
p_{|v|, \phi}(|v|, \phi)=\frac{2|v|}{\pi I^{2}\left(1-|\gamma|^{2}\right)} \exp \left\{\frac{2|\gamma||\nu| \cos \left(\phi-\phi_{0}\right)}{I\left(1-|\gamma|^{2}\right)}\right\} K_{0}\left(\frac{2|\nu|}{I\left(1-|\gamma|^{2}\right)}\right)
$$

where $\mathrm{K}_{0}(\cdot)$ is the modified Bessel function of order zero.

The parameters of the joint PDF of (5) can be determined as follows. $I$ is the geometric mean of the powers of $u_{1}[x, y]$ and $u_{2}[x, y]$, both equal to $P_{m}+P_{a}$. It is therefore equal to $\left(P_{m}+P_{a}\right)$.

$|\gamma|$ and $\phi_{0}$ are the coherence magnitude and the interferometric phase of the interferogram formed from $u_{1}[x, y]$ and $u_{2}[x, y]$. Their expressions can be obtained by equating the expected value of $v[x, y]$, obtained by summing the expected values of the four components in (4) and the expected value of $v[x, y]$, expressed as a function of the parameters of the joint PDF of (5). The sum of the expected values of the components in (4) is given by (note that the expected values of $v_{2}[x, y]$ and $v_{3}[x, y]$ are equal to zero, as $v_{2}[x, y]$ and $v_{3}[x, y]$ are products of uncorrelated random variables)

$$
\begin{aligned}
& E\{v[x, y]\}=E\left\{v_{1}[x, y]\right\}+E\left\{v_{2}[x, y]\right\}+E\left\{v_{3}[x, y]\right\} \\
& +E\left\{v_{4}[x, y]\right\}=E\left\{v_{1}[x, y]\right\}+E\left\{v_{4}[x, y]\right\}= \\
& P_{m}\left|\gamma_{m}\right| \exp \left(j \phi_{0 m}\right)+P_{a}\left|\gamma_{a}\right| \exp \left(j \phi_{0 a}\right)= \\
& P_{m} \sqrt{\left|\gamma_{m}\right|^{2}+\left(\frac{P_{a}}{P_{m}}\right)^{2}\left|\gamma_{a}\right|^{2}+2 \frac{P_{a}}{P_{m}}\left|\gamma_{m}\right| \gamma_{a} \mid \cos \left(\phi_{0 a}-\phi_{0 m}\right)} . \\
& \exp \left(j \arg \left\{\exp \left(j \phi_{0 m}\right)+\frac{P_{a}}{P_{m}} \frac{\left|\gamma_{a}\right|}{\left|\gamma_{m}\right|} \exp \left(j \phi_{0 a}\right)\right\}\right)
\end{aligned}
$$

while the expected value of $v[x, y]$, expressed as a function of the parameters of the joint PDF of (5) is

$$
E\{v[x, y]\}=I|\gamma| \exp \left(j \phi_{0}\right)=\left(P_{m}+P_{a}\right)|\gamma| \exp \left(j \phi_{0}\right)
$$

It therefore holds [3]

$$
\begin{aligned}
& |\gamma|=\left[1+\left(\frac{P_{a}}{P_{m}}\right)\right]^{-1} \cdot \\
& \sqrt{\left|\gamma_{m}\right|^{2}+\left(\frac{P_{a}}{P_{m}}\right)^{2}\left|\gamma_{a}\right|^{2}+2 \frac{P_{a}}{P_{m}}\left|\gamma_{m}\right|\left|\gamma_{a}\right| \cos \left(\phi_{0 a}-\phi_{0 m}\right)} \\
& \phi_{0}=\arg \left\{\exp \left(j \phi_{0 m}\right)+\frac{P_{a}}{P_{m}} \frac{\left|\gamma_{a}\right|}{\left|\gamma_{m}\right|} \exp \left(j \phi_{0 a}\right)\right\}
\end{aligned}
$$

The phase bias resulting from the presence of the azimuth ambiguity is thus given by

$$
\begin{aligned}
& \phi_{\text {bias }}=\phi_{0}-\phi_{0 m}= \\
& \arg \left\{1+\frac{P_{a}}{P_{m}} \frac{\left|\gamma_{a}\right|}{\left|\gamma_{m}\right|} \cos \left(\phi_{0 a}-\phi_{0 m}\right)+j \frac{P_{a}}{P_{m}} \frac{\left|\gamma_{a}\right|}{\left|\gamma_{m}\right|} \sin \left(\phi_{0 a}-\phi_{0 m}\right)\right\}
\end{aligned}
$$

while the phase variance can be related to the coherence magnitude $|\gamma|$ given in (8) by the following formula [5]:

$$
\sigma_{\phi}=\sqrt{\frac{\pi^{2}}{3}-\pi \arcsin (|\gamma|)+\arcsin ^{2}(|\gamma|)-\frac{\mathrm{Li}_{2}\left(|\gamma|^{2}\right)}{2}}
$$

where $\mathrm{Li}_{2}(\cdot)$ is Euler's dilogarithm.

Fig. 2 shows the phase bias, the magnitude of the complex coherence, and the standard deviation of the interferometric phase, as a function of the difference of the interferometric phases $\phi_{0 a}$ $\phi_{0 m}$, for $P_{a} / P_{m}=-5 \mathrm{~dB},\left|\gamma_{m}\right|=0.7$, and $\left|\gamma_{a}\right|=0.6$.

The theoretical results have been validated through simulation and have been shown to be consistent with measurements on TanDEM-X interferograms, affected by azimuth ambiguities [3]. 

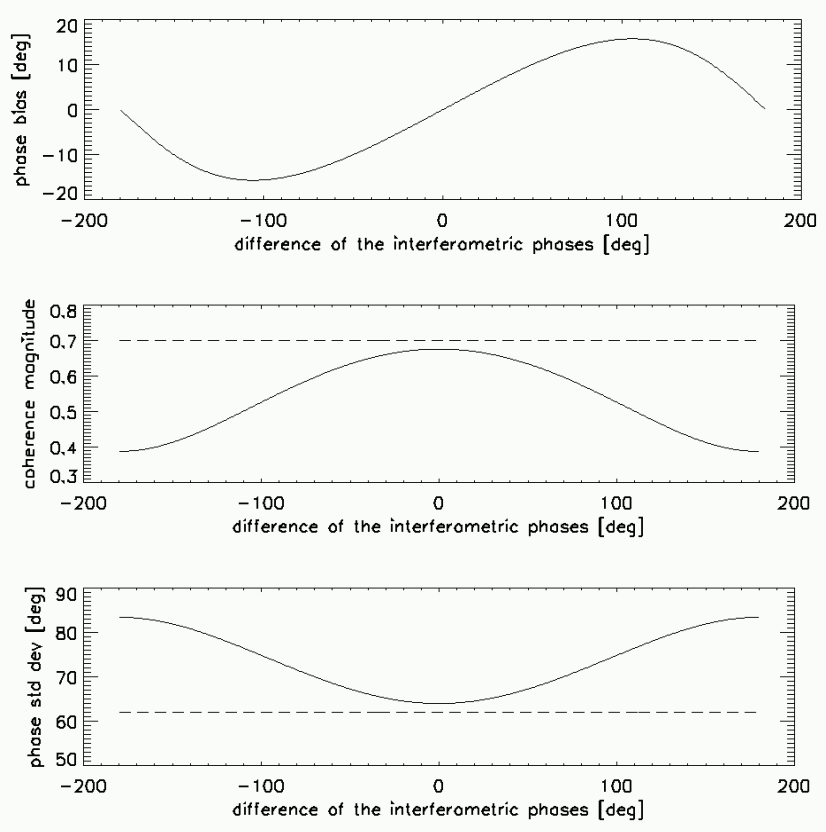

Fig. 2 Phase bias, coherence magnitude and standard deviation of the interferometric phase as a function of the difference of the interferometric phases for $P_{a} / P_{m}=-5 \mathrm{~dB},\left|\gamma_{m}\right|=0.7,\left|\gamma_{a}\right|=0.6$. The dashed lines represent the magnitude of the complex coherence and the standard deviation of the interferometric phase in the absence of ambiguities.

\section{IMPACT OF AZIMUTH AMBIGUITIES ON THE OVERALL SYSTEM PERFORMANCE}

(8) and (10) provide an explanation for the image effects observed in Fig. 1, namely the presence of a shifted replica in the interferometric phase and the modulation of the interferometric coherence.

However, in order to use these results to analyze the impact of azimuth ambiguities on the overall system performance and to understand how to account for azimuth ambiguities in the design of an interferometric system, further manipulations are required.

As apparent from (8) and (10), the local phase bias and the coherence magnitude are dependent on the difference of the interferometric phases $\phi_{0 a}$ and $\phi_{0 m}$, which assumes values comprised between $-\pi$ and $\pi$ (see also Fig. 2). If a probability density function (PDF) is assumed for the difference of these interferometric phases, the expected values and the standard deviations of both the phase bias and the coherence magnitude can be evaluated. In particular, a uniform distribution of the difference of the interferometric phases $\phi_{0 a}-\phi_{0 m}$ over the interval $[-\pi, \pi)$ is assumed. This is a reasonable assumption for the heights of ambiguity of TanDEM-X and terrain with moderate topography.

As far as the phase bias is concerned, it turns out that its expected value is equal to zero

$E\left\{\phi_{\text {bias }}\right\}=\frac{1}{2 \pi} \int_{\theta=-\pi}^{\pi} \arg \left(1+\frac{P_{a}}{P_{m}} \frac{\left|\gamma_{a}\right|}{\left|\gamma_{m}\right|} \exp (j \theta)\right) d \theta=0$

and its standard deviation is given by

$$
\begin{gathered}
\sigma_{\phi_{\text {bias }}}=\sqrt{E\left\{\phi_{\text {bias }}^{2}\right\}-E\left\{\phi_{\text {bias }}\right\}^{2}}=\sqrt{E\left\{\phi_{\text {bias }}^{2}\right\}}= \\
\frac{1}{2 \pi} \sqrt{\int_{\theta=-\pi}^{\pi}\left[\arg \left(1+\frac{P_{a}}{P_{m}} \frac{\left|\gamma_{a}\right|}{\left|\gamma_{m}\right|} \exp (j \theta)\right]^{2} d \theta\right.}
\end{gathered}
$$

Note that $\sigma_{\phi b i a s}$ is a function of $P_{a} / P_{m}$ and $\left|\gamma_{a}\right||| \gamma_{m} \mid$.

As far as the interferometric coherence is concerned, a coherence loss component due to azimuth ambiguities $\left|\gamma_{A m b, A z}\right|$ can be defined as the ratio of the coherence magnitude given in (8) to the coherence magnitude of the ambiguity-free interferogram $\left|\gamma_{m}\right|$

$$
\begin{aligned}
& \left|\gamma_{A m b, A z}\right|=\frac{|\gamma|}{\left|\gamma_{m}\right|}= \\
& {\left[1+\left(\frac{P_{a}}{P_{m}}\right)\right]^{-1} \sqrt{1+\left(\frac{P_{a}}{P_{m}}\right)^{2}\left(\frac{\left|\gamma_{a}\right|}{\left|\gamma_{m}\right|}\right)^{2}+2 \frac{P_{a}}{P_{m}} \frac{\left|\gamma_{a}\right|}{\left|\gamma_{m}\right|} \cos \left(\phi_{0 a}-\phi_{0 m}\right)}}
\end{aligned}
$$

The expected value $E\left\{\left|\gamma_{A m b, A z}\right|\right\}$ and the standard deviation $\sigma_{|\gamma A m b, A z|}$ of the coherence loss component $\left|\gamma_{A m b, A z}\right|$ can be therefore evaluated as a function of $P_{a} / P_{m}$ and $\left|\gamma_{a}\right| /\left|\gamma_{m}\right|$.

The ratio $\left|\gamma_{a}\right| /\left|\gamma_{m}\right|$ can also be expressed as a function of the ratio $P_{a} / P_{m}$ and the signal-to-noise-ratio (SNR) of the ambiguity-free signal $S N R_{m}$. Assuming that the coherence magnitudes $\left|\gamma_{a}\right|$ and $\left|\gamma_{m}\right|$ only differ in the coherence component due to SNR and denoting as $S N R_{a}$ the SNR of the ambiguity signal, we obtain

$$
\begin{aligned}
& \frac{\left|\gamma_{a}\right|}{\left|\gamma_{m}\right|}=\frac{\left|\gamma_{a_{S N R}}\right|}{\left|\gamma_{m_{S N R}}\right|}=\frac{1 /\left(1+S N R_{a}^{-1}\right)}{1 /\left(1+S N R_{m}^{-1}\right)}= \\
& \frac{1 /\left[1+\left(\frac{P_{a}}{P_{m}} S N R_{m}\right)^{-1}\right]}{1 /\left(1+S N R_{m}^{-1}\right)}=\frac{1+S N R_{m}}{\left(\frac{P_{a}}{P_{m}}\right)^{-1}+S N R_{m}}
\end{aligned}
$$

In Fig. 3 the standard deviation of the phase bias $\sigma_{\phi b i a s}$, as well the expected value $E\left\{\left|\gamma_{A m b, A z}\right|\right\}$ and the standard deviation $\sigma_{|\gamma A m b, A z|}$ of the coherence loss component $\left|\gamma_{A m b, A z}\right|$, are displayed as a function of $P_{a} / P_{m}$ and $S N R_{m}$.

As apparent from Fig. 3 (a), the standard deviation of the phase bias $\sigma_{\phi b i a s}$ is strongly dependent on the ratio $P_{a} / P_{m}$ and only weakly dependent on $S N R_{m}$. Fig. 3 (b) instead shows that the average coherence loss component can reach values smaller than 0.63 (which means that the coherence may decrease by almost $40 \%$ due to azimuth ambiguities) for $S N R_{m}$ smaller than $2 \mathrm{~dB}$ and $P_{a} / P_{m}$ approximately equal to $0 \mathrm{~dB}$ (the SNR values are realistic, as the areas affected by azimuth ambiguities are usually characterized by low backscatter and therefore by low SNR). The top-left part of Fig. 3 (b) shows that the coherence loss component can become greater than 1: this is, however, due to the presence of a strong (although coherent) ambiguity signal and is associated with a very significant phase bias (see top-left part of Fig. 3 (a)). Fig. 3 (c) displays the standard deviation of the coherence loss component, which explains the coherence modulation observed in Fig. 1 (right). For $P_{a} / P_{m}$ approximately equal to $0 \mathrm{~dB}$, in fact, not only does the coherence significantly decrease, but its standard deviation is equal to approximately $50 \%$ of the coherence itself. 


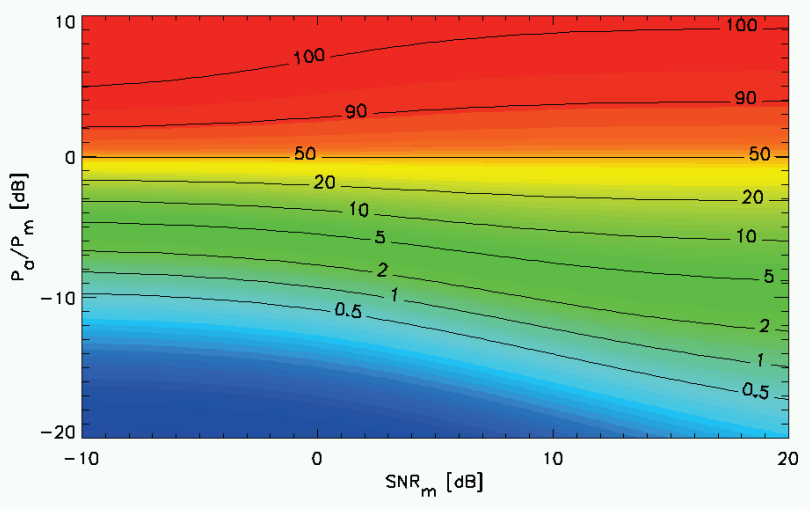

(a)

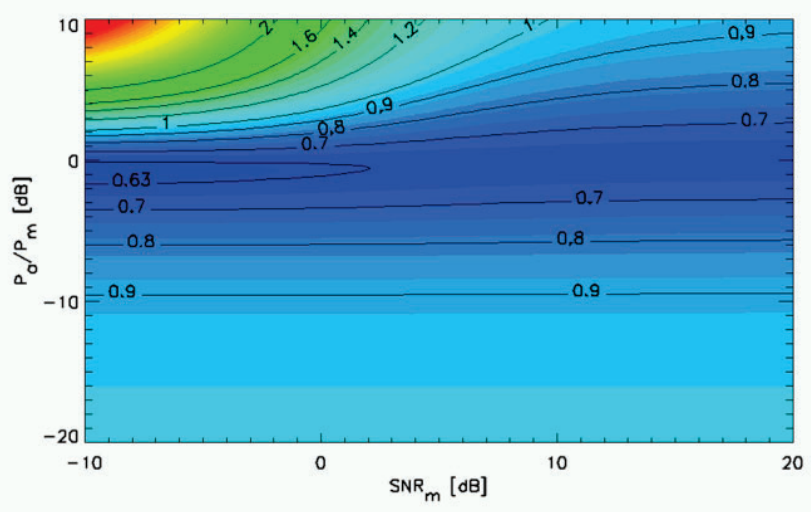

(b)

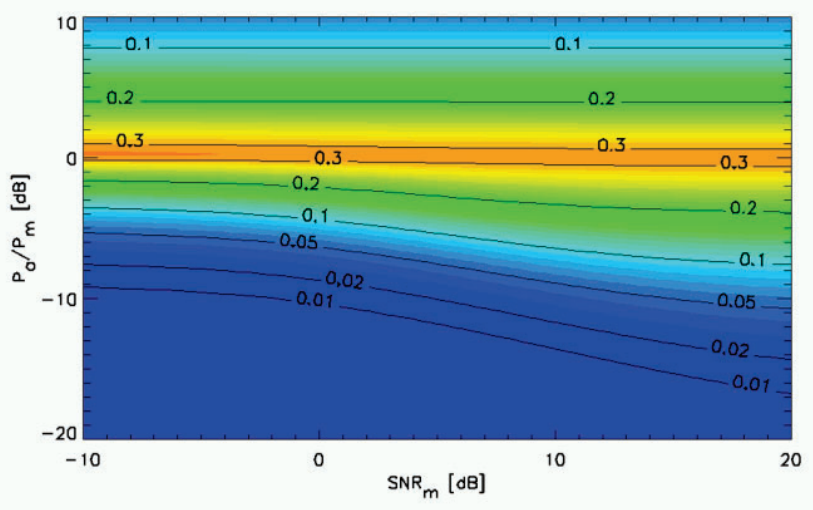

(c)

Fig. 3 (a) Standard deviation of the phase bias. (b) Expected value of the coherence loss component $\left|\gamma_{A m b, A z}\right|$. (c) Standard deviation of the coherence loss component $\left|\gamma_{A m b, A z}\right|$. All parameters are displayed as a function of $P_{a} / P_{m}$ and $S N R_{m}$.

For low values of the ratio $P_{a} / P_{m}$ (e.g. $P_{a} / P_{m}$ smaller than -8 $\mathrm{dB})$, however, the phase bias and the coherence modulation effects become negligible and (1) represents a good approximation of the average coherence loss component due to azimuth ambiguities.

Finally, it is useful to express the ratio $P_{a} / P_{m}$ as

$$
\left(\frac{P_{a}}{P_{m}}\right)_{d B}=F A A S R_{d B}-\left(\frac{\sigma_{a}}{\sigma_{m}}\right)_{d B}
$$

where $\sigma_{a} / \sigma_{m}$ is the ratio of the backscatter of the area affected by the ambiguity to the backscatter of the area responsible for the ambiguity and where FAASR is first azimuth ambiguity-to-signal ratio (FAASR), defined as

$$
F A A S R=\frac{\int_{-B_{p} / 2}^{B_{p} / 2} G^{2}(f+P R F) H^{2}(f) d f}{\int_{-B_{p} / 2}^{B_{p} / 2} G^{2}(f) H^{2}(f) d f}
$$

where $B_{p}$ is the processed Doppler bandwidth, $G^{2}(f)$ is the two-way antenna power pattern in azimuth, $H(f)$ accounts for the amplitude weighting of the Doppler spectrum applied in the processing, and where uniform scene reflectivity is assumed.

A simple example of how to use the plots of Fig. 3 follows. Let us assume a SNR of the ambiguity-free signal $S N R_{m}=10 \mathrm{~dB}$ (this assumption is rather conservative, as the SNR in the areas affected by ambiguities is likely to be lower). Then, a ratio $P_{a} / P_{m}=-10 \mathrm{~dB}$ guarantees a standard deviation of the phase bias smaller than $2^{\circ}$ (see Fig. 3 (a)) and an expected value of the coherence loss component not smaller than 0.9 (see Fig. 3 (b)). Moreover, the standard deviation of the coherence is approximately equal to $5 \%$ of the coherence itself (see Fig. 3 (c) and Fig. 3 (b)), leading to a very slight coherence modulation. In order to achieve a ratio $P_{a} / P_{m}$ smaller than $-10 \mathrm{~dB}$, if a backscatter ratio $\sigma_{a} / \sigma_{m}$ equal to $-17 \mathrm{~dB}$ is assumed, the system has to be designed such that its FAASR is smaller than $-27 \mathrm{~dB}$.

\section{CONCLUSION}

The effects of azimuth ambiguities on interferometric performance (phase bias, coherence modulation) are explained, resorting to the interferogram statistics for jointly circular Gaussian processes. The impact of azimuth ambiguities on the overall system performance is then addressed and plots are provided, which are useful for interferometric performance analysis.

\section{REFERENCES}

[1] F. K. Li and W. T. K. Johnson, "Ambiguities in Spaceborne Synthetic Aperture Radar Systems," IEEE Trans. Aerosp. Electron. Syst., vol. 19, no. 3, pp. 389-397, May 1983.

[2] G. Krieger et al., "TanDEM-X: A Satellite Formation for HighResolution SAR Interferometry," IEEE Trans. Geosci. Remote Sens., vol. 45, no. 11, pp. 3317-3341, Nov. 2007.

[3] M. Villano and G. Krieger, "Impact of Azimuth Ambiguities on Interferometric Performance", IEEE Geosci. Remote Sens. Lett., accepted for publication, $\underline{\mathrm{http}: / / \text { ieeexplore.ieee.org/xpls/abs all.jsp?arnumber }=6164215}$

[4] R. G. Gallager, "Circularly-Symmetric Gaussian Random Vectors,"

http://www.rle.mit.edu/rgallager/documents/CircSymGauss.pdf, retrieved on 21.09.2011.

[5] R. Bamler and P. Hartl, "Synthetic aperture radar interferometry”, Inv. Probl., vol. 14, pp. R1-R54, Feb. 1998. 